\title{
CMOS integrated miniaturized photovoltaic cells for autonomous sensor nodes: simulations and experimental results
}

Andreas Goehlich, Martin Stühlmeyer and Holger Vogt

Fraunhofer Institut für Mikroelektronische Schaltungen und Systeme, Finkenstr. 6147057 Duisburg

Andreas.goehlich@ims.fraunhofer.de

\begin{abstract}
:
Wireless networks for novel applications such as the "internet of things" and "smart dust" require the availability of efficient miniaturized power sources for the realization of autonomous sensor nodes. The most common power source currently utilized is the conventional battery. Energy harvesting from ambient light is an efficient alternative way to circumvent the necessity to periodically replace such batteries. In the frame of a customized development Fraunhofer IMS has developed recently a miniaturized photo cell that can be integrated monolithically on a CMOS-substrate, thus enabling the realization of extremely miniaturized sensor nodes. In order to design and optimize such miniaturized solar cells a careful modelling is required. We report on simulation results on the amorphous solar cells based on experimental material parameters and report on comparisons with experimental results.
\end{abstract}

Key words: CMOS integration, solar cell, amorphous silicon, optical modelling

\section{Introduction}

Wireless networks for novel applications such as the "internet of things" and "smart dust" require the availability of efficient miniaturized power sources for the realization of autonomous miniaturized sensor nodes. The most common power source currently utilized is the conventional battery. Energy harvesting from ambient light is an efficient alternative way to circumvent the necessity to periodically replace such batteries. Integration of miniaturized photo voltaic-cells (PV-cells) in CMOS-substrates with sensors and control circuitry is a promising approach to obtain miniaturized sensor nodes. An additional energy storage element e.g. a super capacitor may be required to ensure continuous energy supply.

In the frame of a customized development project Fraunhofer IMS has developed recently a miniaturized photo cell that can be integrated monolithically on a CMOS-substrate.

In order to design and to optimize such miniaturized integrated solar cells a careful modelling of the integrated PV-cell is required.

In section 2 a short overview on possible integration strategies of PV-cells in CMOS is provided.
Section 3 summarizes the key optical properties of amorphous materials that are needed for optical simulation.

In section 4 simulation results on amorphous silicon solar cells based on experimental and analytically modelled material parameters are reported.

\section{CMOS integration of solar cells}

In the literature several approaches for the integration of PV-cells in CMOS-circuitry are reported. Ferri et al. report on the integration of crystalline silicon PV-cells within bulk-CMOS technology [1]. This approach makes use of the crystalline wafer substrate (bulk silicon) as the crystalline PV-cell, i.e. the "expensive" active silicon area is consumed for the energy harvesting. Another approach makes use of SOI-technology (silicon on insulator) [2]. In this case a crystalline silicon PV-cell is realized by a pn-junction within the handle substrate below the buried oxide of the SOl-wafer. If the backside of the handle substrate is thinned the illumination can be performed from the backside as proposed in [2]. Read out circuitry can be realized then on top of the PV-cell within the thin silicon film. Thus the whole area above the PV-cell can be utilized for the metallization of the CMOS-circuitry. A disadvantage of this approach is connected to the comparatively high cost of a SOI-substrate. An alternative 
approach takes advantage of the post-CMOS integration technique. In this case low temperature PECVD-deposition of amorphous silicon is utilized with a deposition temperature well below $400^{\circ} \mathrm{C}$, which is the approximate limiting temperature required for CMOS compatibility. Such amorphous thin film PVcells are typically build up as a p-i-n diodes, since recombination losses are more pronounced for highly doped amorphous materials. Fundamental work on this approach has been reported previously in ref. [3].

This approach has the additional advantage that a series connection of single PV-cell can be easily achieved in order to realize higher output voltages [3]. The same group has also published another approach for the integration of a CIGS cell (CIGS: copper indium gallium arsenide) [4]. This type of PV-cell is thought to be more efficient as compared to a-Si:H cells, but requires special deposition techniques and materials not available in typical semiconductor fabrication.

Since no active silicon area is consumed by the PV-cell placed on top of the silicon chip and no cost intensive SOI-substrate is necessary, this is the most cost effective approach. Thin film PV-cells exhibit a lower efficiency as compared to crystalline PV-cells in general, however. Basic arrangements of solar cell integration in/on a CMOS substrate are depicted in fig. 1.

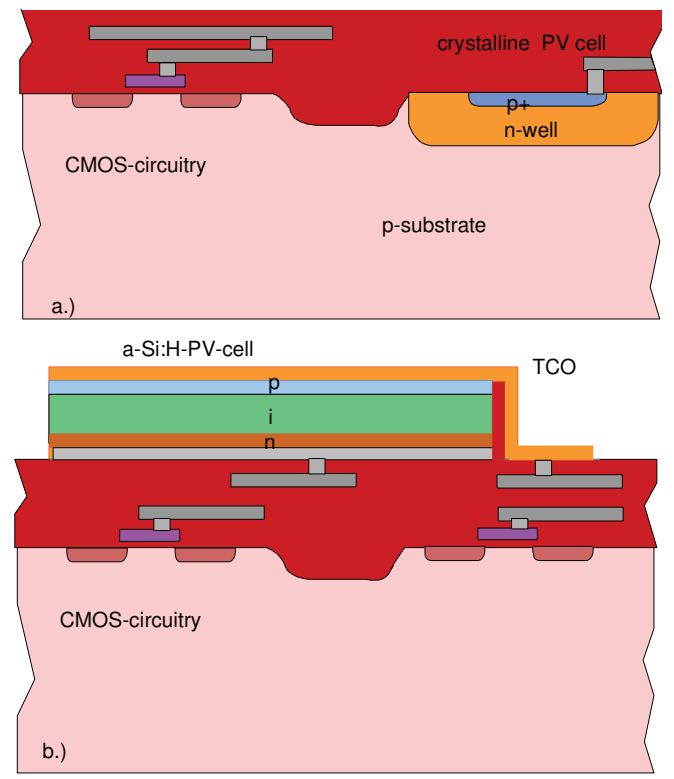

Fig. 1: Schematic sketch of possible integration techniques for PV-cells in/on CMOS (see also refs. [1],[3]). a.) integrated crystalline cell in CMOS. b.) post CMOS integration on top of CMOS.

The "classical" arrangement of a single stage amorphous silicon solar cell is depicted in figure 1b. A p-i-n diode is build up from differently doped amorphous silicon layer-by-layer on a metal electrode that simultaneously serves as a mirror. On top of the p-layer typically a transparent oxide ( $\mathrm{TCO}$, e.g. indium tin oxide (ITO) or aluminum doped tin oxide (AZO)) layer is deposited as the second electrode.

The main restrictions of the post-CMOS integration of PV-cells can be summarized as follows:

a.) A low temperature thermal budget of the post processing steps is required in order not to disturb the underlying CMOS-circuit. This limits the allowed temperature load to about $400{ }^{\circ} \mathrm{C}$.

b.) The utilization of CMOS-compatible materials is required.

c.) The topology of the solar cell stack should not be too high in order to enable subsequent processing e.g. lithography steps. Amorphous silicon and silicon/germanium allow thin layer stacks due to their high absorption in the visible respectively NIR-range.

d.) The post-CMOS integration steps should be feasible with standard semiconductor manufacturing equipment of a typical CMOS/MEMS-fab.

The approach applied in this work for the CMOS-integrated photo cell is based also on an amorphous silicon p-i-n diode (see also fig. 1b). The process developed here is directed to process robustness and stability. The CMOS integration of the solar cell follows the monolithic post-CMOS integration concept by depositing additional layers with the aid of low temperature PECVD-deposition process steps with typical semiconductor manufacturing equipment on top of preprocessed planarized CMOS-substrates [5]. In this way the solar cell can be directly integrated as a power supply in a CMOS-chip that may comprise e.g. power regulation circuitry, a sensor or an actor element and read out circuity. Special attention is attributed to the optimization of the whole layer stack in order to minimize reflection losses on basis of optical modeling that is described in the following sections.

\section{Optical properties of amorphous silicon/germanium materials}

The most important key parameter for the optical modelling of thin film solar cells and photo diodes is the wavelength dependent complex valued refractive index of the amorphous materials.

$$
n(\lambda)=n_{r e}(\lambda)+i n_{\text {im }}(\lambda)
$$


The imaginary part of the refractive index is directly connected to the absorption coefficient $\alpha$ by the relation:

$$
\alpha=4 \pi \frac{n_{i m}}{\lambda}
$$

The optical data can be extracted from ellipsometric data or by theoretical modelling e.g. for development purposes. Ferlauto et al. [6] have provided an analytical semi empirical model of the dielectric function of amorphous silicon and amorphous silicon-germanium compounds, i.e. amorphous layers that are deposited by low temperature PECVD from silane/germane mixtures. The refractive index is connected to the real and imaginary part dielectric function. The adopted model is mainly based on the assumption of a single damped Lorentz-oscillator but taking into account also the Urbach-tail transitions of amorphous materials. The reported formulas allow to calculate the refractive index and absorption coefficient of amorphous a-Si $i_{1-x} \mathrm{Ge}_{\mathrm{x}}: \mathrm{H}$ materials. A set of parameters is provided for different types of materials (optimum/non-optimum material), that depends linearily on the optical band gap. An optical band-gap around $E_{\text {gap }}=1.7$ $\mathrm{eV}$ corresponds to amorphous silicon while doping with germanium leads to a lowering of the band gap. The calculated refractive indices of amorphous $\mathrm{Si}_{x} \mathrm{Ge}_{1-x}: \mathrm{H}$ based on the model communicated by Ferlauto et al. are depicted in figure 2 for "optimized "material ("material l" see ref. [6]) and for different values of the optical band gap. A smaller bandgap corresponds to a higher germane fraction. Thus doping of amorphous silicon with germane leads to an enhancement of the red response. This can be advantageously used in stacked tandem or triple PV-cells in order to enhance the energy harvesting of the red and NIR-spectral range.

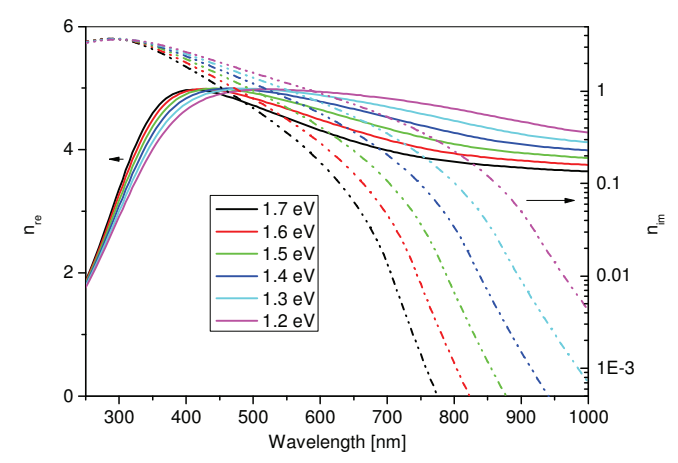

Fig. 2: Calculated refractive index according parameters presented in ref. [6] for optimized a-Si $i_{-x} G e_{x}: H$ material for different values of the optical gap.

\section{Optical and electrical Simulations}

A well known simulation technique for one dimensional optical interference problems of multiple thin film layer stacks is the so called transfer matrix method. It is described in detail in several textbooks e.g. in ref. [7], therefore the approach is only shortly sketched here. The method is based on the continuity of the tangential component of the electrical and magnetic field of a plane electromagnetic wave at the interface between adjacent optical materials. A complex valued $2 \times 2$ matrix couples the electrical and magnetic field amplitudes entering and exiting each dielectric layer.

These matrices are multiplied for each layer to obtain the overall system matrix. The complex valued reflection and transmission amplitudes as well as the real valued intensity related reflectance and transmission coefficients are obtained from the elements of the system matrix by formulas e.g. presented in ref. [7].

The field at each interface can be calculated iteratively backward starting with the amplitude of the transmitted field at the exit layer (calculated with the aid the previously mentioned transmission amplitude coefficient) and multiplying with the corresponding last transfer matrix. The electric and magnetic fields $\mathrm{u}_{\mathrm{i}}(\mathrm{z}), \mathrm{v}_{\mathrm{i}}(\mathrm{z})$ inside each layer (with normal in $\mathrm{z}$ direction) are decomposed in a forward and a backward running wave, e.g.:

$$
u_{i}(z)=\alpha_{i} e^{i k_{i} z}+\beta_{i} e^{-i k_{i} z}
$$

$k_{i}$ is the component of the wave vector along the z-axis. The complex amplitudes $\alpha_{i}, \beta_{i}$ are determined by the previously calculated interface fields at the entrance and exit interface of each layer $i$. The spatially resolved normalized energy flux (i.e. the normalized Poynting vector $S(z)$ ) and the differential absorption (i.e. the negative spatial derivative of the energy flux $d S / d z$ ) inside each layer is then obtained from the electrical and magnetic fields. For normal incidence the differential absorption can be written as:

$$
\begin{aligned}
-\frac{d S}{d z} & =2 k_{0} \frac{n_{r e}^{(i)} n_{i m}^{(i)}}{n_{0}} U(z) \\
U & =\left(\alpha_{i}^{*} e^{-i k_{i}^{*} z}+\beta_{i}^{*} e^{i k_{i}^{*} z}\right)\left(\alpha_{i} e^{i k_{i} z}-\beta_{i} e^{-i k_{i} z}\right)
\end{aligned}
$$

Where $\mathrm{k}_{\mathrm{i}}$ and $\mathrm{n}_{\mathrm{i}}$ denote the wave vector and the refractive index of the respective media.

The differential absorption can be transformed into the optical generation rate by the relation:

$$
G_{o p t}(z)=-1 / h c \int_{\lambda_{1}}^{\lambda_{2}} d \lambda \lambda I_{\lambda}(\lambda) \frac{d S}{d z}(\lambda)
$$

$\mathrm{I}_{\lambda}$ is the excitation spectrum such as the AM 1.5 reference distribution in $\mathrm{W} \mathrm{cm}^{-2} \mathrm{~nm}^{-1}$. 
The integration is performed over the sensitive wavelength range of the PV-cell. This generation rate then can be coupled to electrical device simulation. A computer code has been established that calculates on basis of the aforementioned considerations angle and wavelength dependent optical parameters such as the spatial dependencies of the energy flux, the differential absorption and the optical generation rate. The code allows the utilization of measured tabulated data, as well as data based on the analytical model according to ref. [6]. As an application the spectrally resolved absorption within the intrinsic layer of an a-Si single PV-cell is depicted in the figure $3 a$ for different thicknesses of the TCO layer. In this case indium tin oxide (ITO) has been assumed as the top electrode material. Refractive index data were taken from ref. [8]. As the intrinsic layer amorphous silicon with $400 \mathrm{~nm}$ thickness and a refractive index according to the analytical model ref. [6] with an optical gap of $1.7 \mathrm{eV}$, which is a typical value for a-Si: $\mathrm{H}$, has been selected. Measured values of the refractive index have been used for the $p$ and $n$ doped-regions of the pin diode. For broad band illumination with an excitation spectrum $I_{\lambda}(\lambda)$ the short circuit current $J_{S C}$ of optical active layer $\mathrm{j}$ can be determined:

$$
J_{s c}^{(j)}=\frac{e}{h c} \int_{\lambda_{1}}^{\lambda_{2}} d \lambda \lambda a_{j}(\lambda) \eta_{\mathrm{int}}^{(j)}(\lambda) I_{\lambda}(\lambda)
$$

with $a_{j}(\lambda)$ denoting the absorbance within the optically active layer $j$ (e.g. the intrinsic layers of stacked cells) and $\eta^{(j)}$ int denotes the internal conversion efficiency that accounts for the conversion of an absorbed photon to a collected charge reaching the electrodes.
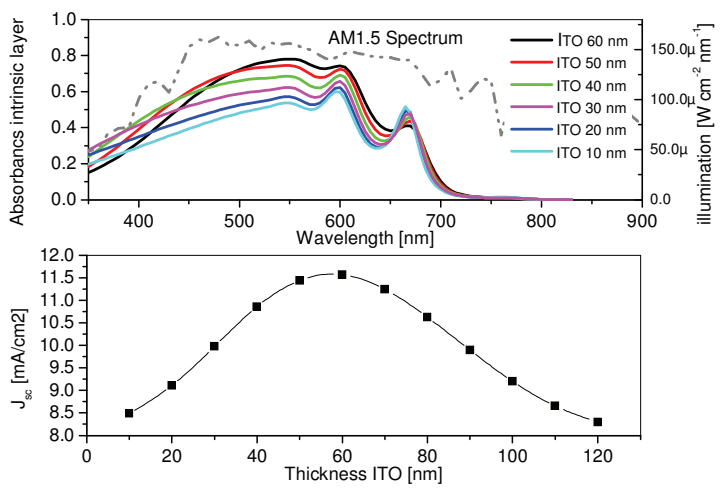

Fig. 3: (a.) Calculated spectrally resolved absorbance of intrinsic layer for a $400 \mathrm{~nm}$ thick $P V$-cell for different thicknesses of the TCO. Normal incidence is assumed.( b.) Calculated short circuit current for $\eta_{\text {int }}=1$ (see eq. 6).

As a first estimation $\eta^{(j)}{ }_{\text {int }} \approx 1$ can be used. In this approximation the short circuit current density can be estimated on basis of optical data only.
In the figure $3 \mathrm{~b}$ the maximum current density is plotted as a function of the TCO-thickness. Illumination with a reference spectrum AM 1.5 with $100 \mathrm{~mW} / \mathrm{cm}^{-2}$ and perpendicular incidence has been assumed for the calculation of $\mathrm{J}_{\mathrm{sc}}$. The spectral integration has been performed from $350-850 \mathrm{~nm}$. It exhibits a maximum for the ITO-layer thickness at $60 \mathrm{~nm}$, indicating an optimum value for the antireflection layer. The calculated spectrally resolved absorption can be compared to quantum efficiency (QE) measurements. Here miniaturized PV-cell test structures with area approx. $4 \mathrm{~mm}^{2}$ manufactured with a-Si:H and a-SiGe:H intrinsic layers have been generated. An approx. $60 \mathrm{~nm}$ thick ITO-layer has been deposited as the TCO on the a-Si:H cell. For comparison the calculated spectrally dependent absorption of the intrinsic layer only is plotted. The refractive index for the intrinsic layer (width $400 \mathrm{~nm}$ ) has been chosen according to the model of ref [6.] with an optical gap of $1.7 \mathrm{eV}$. Only the amplitude of the calculated absorption profile has been multiplied by a factor of 0.8 to fit the measured external quantum efficiency (fig. 4a).
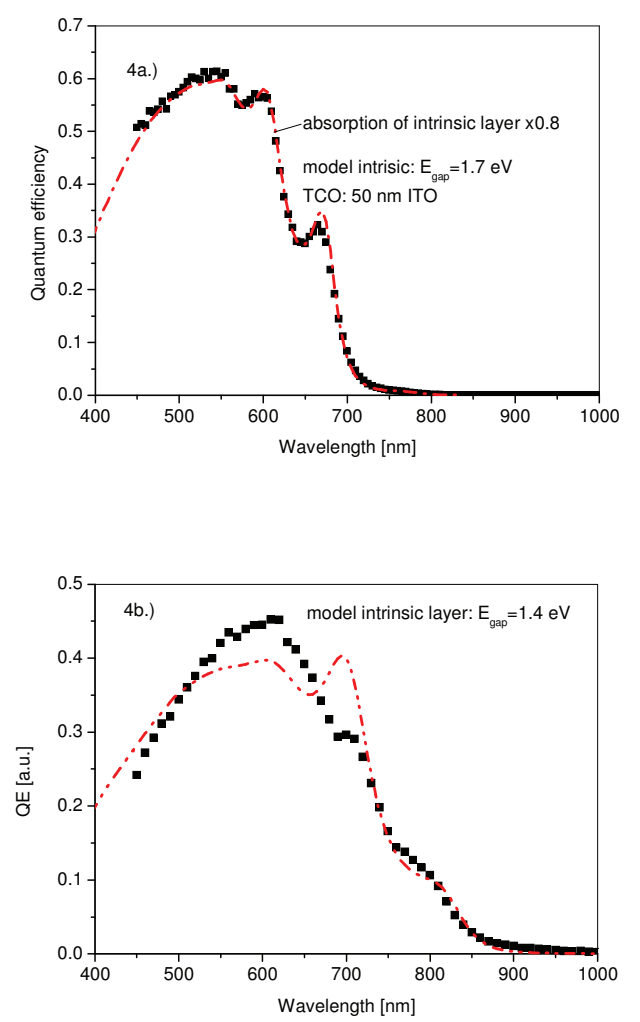

Fig. 4: Comparison of spectrally resolved $Q E$ measurement with the simulated absorbance: (a.) aSi:H cell intrinsic layer and (b.) of a germane doped sample (note: scale is in arbitrary units in b.).

This can be indicative for additional collectionor scattering-losses.

While good agreement with the optical calculation is found for the pure a-Si:H test 
sample, some differences are observed for a germane doped test sample (fig. 4b.).The refractive index according ref. [6] with $E_{\text {gap }}=1.4$ $\mathrm{eV}$ has been used in this calculation. The red shift of the spectral response for the germane doped sample is clearly observed. The deviations with respect to the optical simulation may be attributed to transport effects that are pronounced in doped materials.

Examples of the calculated optical generation profiles for AM 1.5 illumination are depicted in figure 5 for a single and a tandem cell, both deposited on an aluminum mirror as the reflector. The observed oscillations are due to interference effects. For the tandem cell the width of the bottom cell has been chosen to fulfill the current matching condition of both active layers.

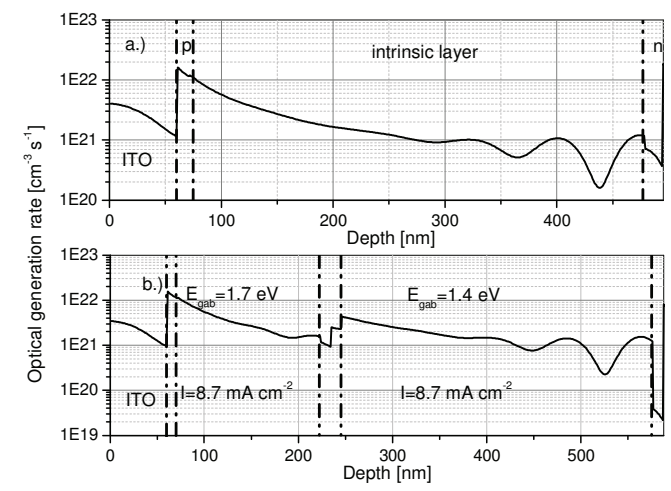

Fig. 5: Example of a calculation of the optical generation rate for a single (a.) and a tandem cell (b.) for AM 1.5 illumination and normal incidence. For the intrinsic layers the model according ref. [6] has been adopted.

The calculated generation rate can be coupled to device simulations such as SDEVICE from Synopsis. In the following example a one dimensional calculation of the amorphous single a-Si $\mathrm{p}$-i-n-structure is presented. In contrast to crystalline silicon the band gap distribution of states is considerably more complex for amorphous materials. The distribution can be described by exponential band gap tails extending from the conduction and valence band respectively and by two identical near mid-gap Gaussian distributions for amphoteric acceptor and donor dangling bond states separated by a correlation energy $[9,10]$. The main material data used here where based on the data from ref. [10] and where adjusted to approximately fit the measured short circuit current.

A comparison of a simulation of the IVcharacteristics with the measurement of a PVtest structure obtained at AM1.5 illumination is made in figure 6 .
The generation rate has been calculated with the established computer code and has been scaled to the measured quantum efficiency (see figure $4 \mathrm{a}$ ). A series resistance of $0.75 \mathrm{Ohm}$ has been assumed in the calculation. The simulated IV-characteristics is consistent with the measurement.

The experimentally extracted parameters of this test cell with an ITO-TCO amount to about $V_{O C}=0.77 \mathrm{~V}$, fill factor of $66 \%$ and an efficiency of about $4.8 \%$ at AM 1.5 illumination and are approximately met by the simulation setup.

The simulation setup allows to get insight in the microscopic carrier generation processes.

The distribution of the carrier current densities, the free carrier densities and the electric field is depicted in figure 7 at $0.65 \mathrm{~V}$ forward bias (near maximum power point) of the diode.

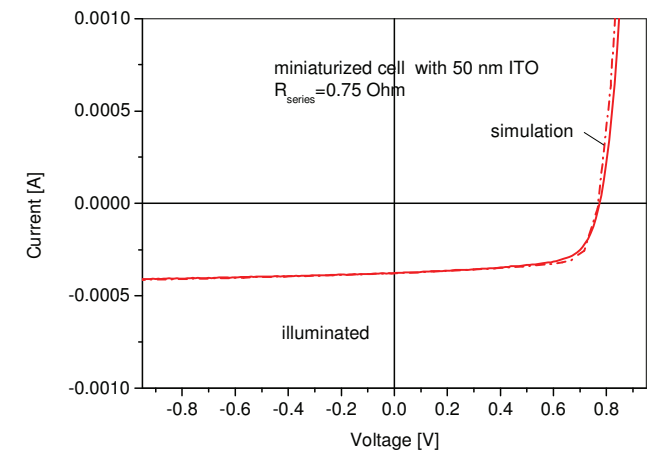

Fig. 6: Comparison of the experimental (solid) and simulated (dash-dotted) IV-characteristics for AM1.5 illumination. For the refractive index of the intrinsic layer the model according ref. [6] has been adopted.

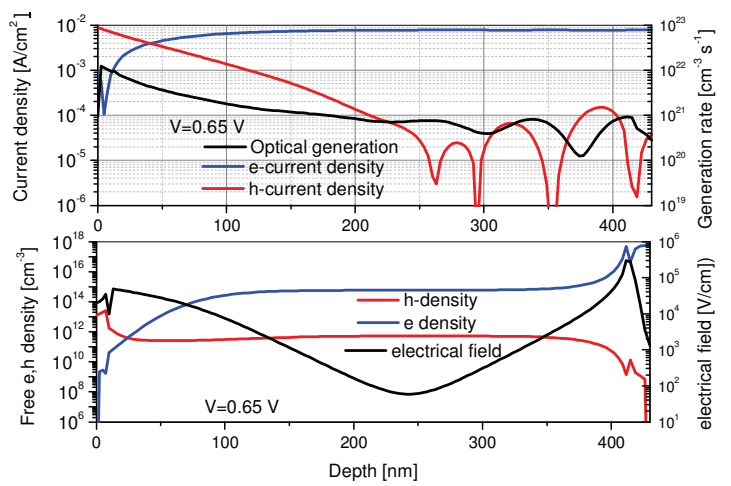

Fig. 7: Calculated internal fields and densities at $V=0.65 V$ and $A M 1.5$ illumination. The light enters from the left side through the $p^{+}$-layer.

It is observed that the hole current follows the optical excitation and is concentrated near the $\mathrm{p}^{+}$-entrance layer, while the electrons -due to their higher mobility- are distributed over nearly the whole intrinsic layer. The electrical field exhibits a minimum shifted to the $\mathrm{n}^{+}$-electrode. 
On basis of this simulation setup parameter variations of the geometry can be performed. In Figure 8 the variation of the width of the intrinsic layer. For each layer width the optical generation rate has been calculated on the grid of the electrical simulation und coupled to the electrical simulation as an external source. The calculated efficiency exhibits a flat maximum around $200 \mathrm{~nm}$. For larger values of the intrinsic layer width the fill factor of the cell slowly decreases.

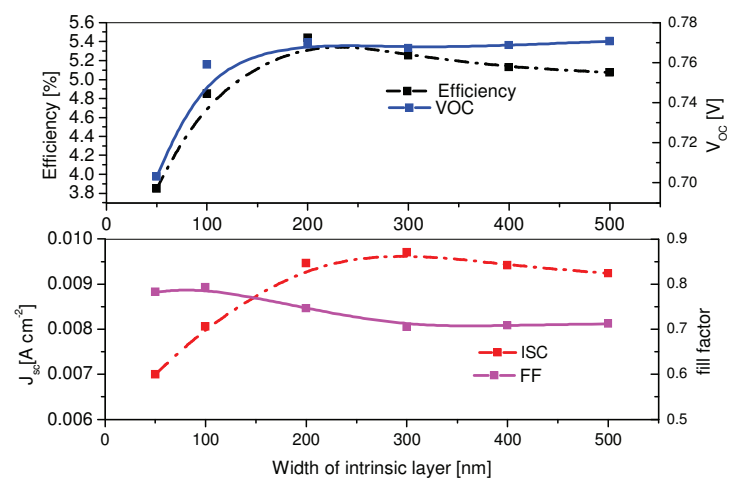

Fig. 8: Calculated dependence of efficiency, open voltage, short circuit current and fill factor on the width of the intrinsic layer for AM1.5 illumination. The inserted lines are intended to guide the eye.

\section{Summary}

In this contribution we have reported on optical and electrical simulations for miniaturized amorphous silicon solar cells. The analytic model according Ferlauto et al. [6] for the refractive index of amorphous silicon has been proved to be suitable for the optical modelling. A computer code has been established that calculates the absorbance, short circuit current and the optical generation rate for amorphous single and tandem cells. The calculated generation rates can be coupled as external generation rates to more sophisticated simulation tools in order to speed up calculation time.

A good agreement has been found in particular for the calculated relative spectral shape of the absorbance of the intrinsic layer with the measured quantum efficiency of a miniaturized a-Si:H test cell with an ITO-TCO-layer. On basis of the deduced generation rate an electrical simulation has been performed that can describe the measured IV-characteristics. The open voltage and the efficiency is in good agreement with the simulation. A parameter variation of the width of the cell has been performed that exhibits a flat maximum at around $200 \mathrm{~nm}$.

However due to the number of required microscopic parameters, which are still not precisely known, further effort is necessary to improve the reliability of the simulation.

\section{References}

[1] M. Ferri, D.Pinna, M.Grassi et al.: "Model of Integrated Micro Photovoltaic Cell Structures for Harvesting Supplied Microsystems in 0.35- $\mu \mathrm{m}$ CMOS Technology" in IEEE Sensors 2010 conference, p.232, 2010

[2] O. Bulteel, R. Delamarre and D. Flandre,"High-efficiency solar cell embedded in SOI substrate for ULP autonomous circuits" in IEEE SOI conference 2009, Foster City,CA/USA,2009

[3] J. Lu, A.Y.Kovalgin, K.H.M. v.d.Werf et al.: "Integration of Solar Cells on Top of CMOS Chips Part I: a-Si Solar Cells" in IEEE TRANSACTIONS ON ELECTRON DEVICES, VOL. 58, NO. 7, JULY 2011,pp. 2014

[4] J. Lu, W.Liu, A.Y.Kovalgin et al.: "Integration of Solar Cells on Top of CMOS Chips Part II: CIGS Solar Cells" in IEEE TRANSACTIONS ON ELECTRON DEVICES, VOL. 58, NO. 8, August 2011, pp. 2620

[5] M. Stühlmeyer; A. Goehlich, H. Vogt: "Customized development of a CMOS compatible solar cell for autonomous sensor nodes" In: Fraunhofer-Institut für Mikroelektronische Schaltungen und Systeme : Fraunhofer-Institut für Mikroelektronische Schaltungen und Systeme <Duisburg > : Annual report. Duisburg 2014, pp. 56 - 58.

[6] A.S. Ferlauto, G.M.Ferreira, J.M.Pearce et al.: "Analytical model for the optical functions of amorphous semiconductors from the near infrared to ultra violet: Applications in thin film photovoltaics", J.Applied.Phys, Vol.92,No.5, 2426 (2002).

[7] G.R.Fowles, "Introduction to Modern Optics", $2^{\text {nd }}$. Ed., Dover Publications, N.Y. (1989)

[8] http://www.sspectra.com/sopra.html

[9] N. Palit,U.Dutta, P. Chatterjee,"Detailed computer modeling of Semiconductor devices", Indian J. Phys, 11-35 (2006)

[10] P.Otero, A.Roriguez, M.Vetter et al.: "Simulation of the temperature characteristics of a-Si:H solar cell current voltage characteristics", Proc. $8^{\text {th }}$ Spanish Conference on electronic devices, CDE 2011. 